\title{
Avaliação clínica de pacientes com úlceras de perna acompanhados em ambulatório
}

\author{
Clinical evaluation of patients with leg ulcers attended in outpatient clinic \\ Evaluación clínica de pacientes con úlceras de piernas en tratamiento ambulatorio
}

\begin{abstract}
Fernanda Rabello Sergio ${ }^{1}$ (]) Isabelle Andrade Silveira ${ }^{1}$ (]) Beatriz Guitton Renaud Baptista de Oliveira² (1)
\end{abstract}

${ }^{1}$ Universidade Federal Fluminense, Escola de Enfermagem Aurora de Afonso Costa, Programa Acadêmico em Ciências do Cuidado em Saúde. Niterói, RJ, Brasil

2 Universidade Federal Fluminense, Escola de Enfermagem Aurora de Afonso Costa. Niterói, RJ, Brasil.
Autor correspondente:

Fernanda Rabello Sergio

E-mail: fernandarabello1105@gmail.com

Recebido em 01/05/2020.

Aprovado em 21/07/2020.

DOI:https://doi.org/10.1590/2177-9465-EAN-2020-0139

\section{RESUMO}

Objetivo: realizar avaliação clínica e sociodemográfica de pacientes com úlceras de perna. Método: estudo transversal quantitativo, com 105 pacientes com úlceras de perna em ambulatórios da rede pública de Niterói/RJ, aprovado pelo Comitê de Ética em Pesquisa da Faculdade de Medicina da Universidade Federal Fluminense. Os dados foram coletados no período de janeiro a agosto de 2019, analisados por estatística descritiva e dispostos em tabelas. Resultados: pacientes do sexo masculino (57,1\%), de 60 a 80 anos (60\%), com ensino fundamental incompleto (45,7\%), renda de até 1 salário mínimo (64,8\%) A maioria das lesões foi de etiologia venosa $(76,2 \%)$, de tempo igual ou superior a 40 meses $(54,3 \%)$, com tamanho maior que $10 \mathrm{~cm}^{2}(53,3 \%)$, apresentando exsudato seroso $(91,4 \%)$ em pequena quantidade $(40 \%)$ e predominância de tecido granulado $(36,2 \%)$. A dor foi um achado frequente, relacionada com a posição do membro (31,4\%). Conclusão e implicações para a prática: o conhecimento do perfil da população acompanhada e das características clínicas das lesões favorece a otimização do tratamento, permitindo um planejamento específico da assistência de enfermagem.

Palavras-chave: Úlcera; Úlcera da perna; Ferimentos e lesões; Enfermagem; Assistência ambulatorial.

\section{ABSTRACT}

Objective: to perform clinical and sociodemographic evaluation of patients with leg ulcers. Method: cross-sectional study with a quantitative approach, performed with 105 patients with leg ulcers in public outpatient clinics located in Niterói/RJ, approved by the Research Ethics Committee of the Medical School of Universidade Federal Fluminense. Data were collected from January to August 2019, analyzed by descriptive statistics and organized in tables. Results: most were male patients (57.1\%), aged between $60-80$ years $(60 \%)$, with incomplete primary education (45.7\%) and monthly income up to 1 minimum wage $(64.8 \%)$. Most ulcers were of venous etiology $(76.2 \%)$, present for 40 months or more $(54.3 \%)$, with size greater than $10 \mathrm{~cm}^{2}(53.3 \%)$, having serous exudate $(91.4 \%)$ in small quantity $(40 \%)$ and predominance of granulation tissue $(36.2 \%)$. Pain was a frequent finding, related with the leg position (31.4\%). Conclusion and implications for practice: the knowledge of the population profile and clinical characteristics of wounds favors treatment optimization, allowing specific nursing care planning.

Keywords: Ulcer; Leg ulcer; Wounds and Injuries; Nursing; Ambulatory Care.

\section{RESUMEN}

Objetivo: realizar evaluación clínica y sociodemográfica de pacientes con úlceras de piernas. Método: estudio transversal, cuantitativo, con 105 pacientes con úlceras de piernas en clínicas públicas en Niterói/RJ, aprobado por el Comité de Ética en Investigación de la Facultad de Medicina de la Universidade Federal Fluminense. Los datos fueron recolectados de enero a agosto de 2019, analizados por estadística descriptiva y presentados en tablas. Resultados: pacientes del sexo masculino (57.1\%), de 60 a 80 años (60\%), con educación primaria incompleta (45.7\%), ingresos de hasta 1 salario mínimo (64.8\%). La mayoría de las lesiones fueron de etiología venosa $(76,2 \%)$, con una duración de 40 meses o más $(54,3 \%)$, con un tamaño superior a $10 \mathrm{~cm}^{2}$ $(53,3 \%)$, presentando exudado seroso $(91,4 \%)$ en pequeña cantidad $(40 \%)$ y predominio de tejido granulado $(36,2 \%)$. El dolor fue un síntoma frecuente, relacionado con la posición del miembro $(31,4 \%)$. Conclusión e implicaciones para la práctica: el conocimiento del perfil de la población monitoreada y las características clínicas de las lesiones favorecen la optimización del tratamiento, lo que permite una planificación específica de la atención de enfermería.

Palabras clave: Úlcera; Úlcera de la Pierna; Heridas y Traumatismos; Enfermería; Atención Ambulatoria. 


\section{INTRODUÇÃO}

Diante das mudanças no perfil epidemiológico e demográfico populacional, com aumento da expectativa de vida, podemos prever um aumento no número de indivíduos com doenças crônico-degenerativas decorrentes do envelhecimento. ${ }^{1,2}$ Dessa forma, com o consequente aumento global de doenças crônicas, estima-se uma elevação concomitante na quantidade de indivíduos com lesões secundárias à patologia de base. ${ }^{3}$

A presença de comorbidades como diabetes mellitus, hipertensão arterial sistêmica, obesidade, neoplasias e vasculopatias favorece o desenvolvimento de feridas complexas, de difícil cicatrização. ${ }^{2}$ As lesões crônicas associadas a essas comorbidades apresentam retardo no processo de cicatrização, ocasionando importante impacto econômico aos serviços de saúde, pois demandam atendimento interdisciplinar especializado, uso de produtos específicos e longo período de tratamento. ${ }^{2,4}$ Além disso, causam implicações importantes na qualidade de vida e situação econômica do indivíduo e de sua família, uma vez que ocasionam o afastamento das atividades laborais e dificultam a realização de atividades cotidianas. ${ }^{1,2}$

A avaliação do enfermeiro, incluindo as orientações pertinentes e escolha do tratamento mais adequado deve ser feita com base nas características das lesões e presença de comorbidades, assim como no perfil dos pacientes e suas condições sociodemográficas e econômicas. ${ }^{2}$

Dessa forma, evidencia-se a importância do conhecimento das características socioeconômicas e clínicas dos pacientes acompanhados através de assistência ambulatorial, visando identificação de seu perfil e avaliação de possíveis modificações necessárias para organização do serviço, contribuindo com a orientação e planejamento de metas mais eficazes, permitindo um planejamento do cuidar pelo enfermeiro condizente com a realidade local e direcionada às necessidades da clientela. ${ }^{2-5}$

O processo de cicatrização de feridas agudas compreende uma série de etapas bem entendidas pela ciência e acontece de forma ordenada, conforme a cicatrização ocorre. Quando se trata de uma ferida crônica, esse processo se encontra interrompido ou desordenado. Dessa forma, torna-se necessário mais estudos para melhor entendimento dos fatores envolvidos na fisiopatologia das feridas crônicas. ${ }^{6}$

Esse processo complexo ocorre normalmente em fases que se sobrepõe. Inicialmente, há a hemostasia e a fase inflamatória, quando ocorre aumento da permeabilidade vascular, quimiotaxia e ativação celular. Em seguida, tem início a fase proliferativa, com migração de fibroblastos, produção de tecido de granulação e angiogênese. A fase de remodelação pode levar de semanas a anos e é caracterizada pela deposição de colágeno. ${ }^{4,6}$ Diversos fatores podem influenciar o processo de cicatrização, incluindo tamanho da ferida, profundidade, localização, idade e presença de comorbidades. ${ }^{6}$

Diante desse contexto, este estudo teve como objetivo realizar avaliação clínica e sociodemográfica de pacientes com úlceras de perna acompanhados em ambulatório.

\section{METODOLOGIA}

Trata-se de um estudo observacional, transversal, de abordagem quantitativa, realizado através do atendimento de 105 pacientes com úlceras de perna acompanhados no Ambulatório de Reparo de Feridas de um Hospital Universitário no estado do Rio de Janeiro/RJ e em uma Policlínica Regional/RJ, ambas unidades da rede pública da cidade de Niterói.

Este estudo utilizou a amostragem em sequência, recrutando os pacientes de forma consecutiva durante a assistência ambulatorial de enfermagem de rotina, sendo incluídos no estudo pacientes com úlceras de perna com idade acima de 18 anos e excluídas as participantes com gravidez suspeita ou confirmada. A coleta dos dados foi realizada pela enfermeira pesquisadora responsável pelos atendimentos, no período de janeiro a agosto de 2019, utilizando o instrumento Leg Ulcer Measurement Tool (LUMT), que engloba itens de avaliação clínica da úlcera, dor, qualidade de vida e é considerado adequado para uso no contexto da realidade brasileira. ${ }^{7} \mathrm{~A}$ avaliação da área da lesão foi realizada através do decalque das úlceras em acetato estéril para utilização da técnica de planimetria manual.

As variáveis de caracterização sociodemográfica e histórico clínico foram: sexo, idade, escolaridade, renda e comorbidades presentes. As variáveis de caracterização da lesão foram: etiologia da lesão, localização, tempo de úlcera, tamanho da úlcera em cm², tipo e quantidade de exsudato, quantidade de tecido necrótico e de tecido de granulação, presença e tipo de edema, frequência e intensidade da dor e percepção da qualidade de vida.

A pesquisa foi aprovada pelo Comitê de Ética em Pesquisa da Faculdade de Medicina da Universidade Federal Fluminense, respeitando os princípios estabelecidos na Resolução $n^{\circ} 466 / 2012$ do Conselho Nacional de Saúde (CNS) com o CAAE $n^{\circ}$ 56252216.2.0000.5243.

Os participantes foram convidados a participar da pesquisa, esclarecidos quantos aos objetivos e método do estudo e a coleta de dados ocorreu após assinatura do Termo de Consentimento Livre e Esclarecido (TCLE).

Os dados coletados, a partir dos instrumentos de pesquisa, foram dispostos em uma planilha eletrônica do programa Microsoft Excel e posteriormente tabulados em relação às características sociodemográficas e dados clínicos e descritivos das lesões.

\section{RESULTADOS}

A amostra foi composta por um total de 105 participantes, sendo a maioria do sexo masculino $(57,1 \%)$, com idade entre 60 e 80 anos (60\%), apresentando como comorbidades mais frequentes a insuficiência venosa crônica ( $81 \%$ ) e a hipertensão arterial sistêmica (73,3\%). Quanto à etiologia das lesões, a maioria identificada foi de úlceras venosas (76,2\%) (Tabela 1).

Em relação ao grau de escolaridade, a maioria declarou possuir ensino fundamental incompleto $(45,7 \%)$, seguido de ensino médio completo $(23,8 \%)$, ensino fundamental completo $(11,4 \%)$, analfabetos $(5,8 \%)$, ensino médio completo e ensino superior completo (ambos representando 4,8\%) e ensino 
Tabela 1. Distribuição dos pacientes segundo sexo, idade, presença de comorbidades e tipo de úlcera. Niterói, RJ, 2019. $(\mathrm{N}=105)$.

\begin{tabular}{lcc}
\hline \multicolumn{1}{c}{ Variável } & N & $\%$ \\
\hline Sexo & 45 & 42,9 \\
\hline Feminino & 60 & 57,1 \\
\hline Masculino & & \\
\hline Idade (anos) & 3 & 2,9 \\
\hline $20 \mid-40$ & 25 & 23,8 \\
\hline $40 \mid-60$ & 63 & 60,0 \\
$60 \mid-80$ & 14 & 13,3 \\
\hline $80 \mid-100$ & & \\
\hline Comorbidades & 77 & 73,3 \\
\hline Hipertensão Arterial Sistêmica & 85 & 81,0 \\
\hline Insuficiência Venosa Crônica & 46 & 43,8 \\
\hline Diabetes Mellitus & 5 & 4,8 \\
\hline Doença Arterial Obstrutiva & & \\
Periférica & & \\
\hline Tipo de úlcera & 80 & 76,2 \\
\hline Venosa & 20 & 19,0 \\
\hline Diabética & 1 & 1,0 \\
\hline Arterial & 4 & 3,8 \\
\hline Mista & &
\end{tabular}

Tabela 2. Distribuição das úlceras segundo localização, tempo e tamanho. Niterói, RJ, 2019. ( $\mathrm{N}=105$ ).

\begin{tabular}{|c|c|c|}
\hline Variável & $\mathbf{N}$ & $\%$ \\
\hline \multicolumn{3}{|l|}{ Localização da úlcera } \\
\hline Máleolo & 59 & 56,2 \\
\hline Terço Anterior Distal & 8 & 7,6 \\
\hline Terço Posterior Distal & 6 & 5,7 \\
\hline Terço Distal & 4 & 3,8 \\
\hline Dorso do pé & 10 & 9,5 \\
\hline Coto do membro & 9 & 8,6 \\
\hline Halux & 6 & 5,7 \\
\hline Região plantar do pé & 3 & 2,9 \\
\hline \multicolumn{3}{|c|}{ Tempo de úlcera (meses) } \\
\hline $0 \mid-6$ & 23 & 21,9 \\
\hline $6 \mid-11$ & 5 & 4,8 \\
\hline $11 \mid-21$ & 7 & 6,7 \\
\hline $21 \mid-31$ & 8 & 7,6 \\
\hline $31 \mid-40$ & 5 & 4,8 \\
\hline Maior ou igual a 40 & 57 & 54,3 \\
\hline \multicolumn{3}{|l|}{ Tamanho da Úlcera } \\
\hline$<2.5 \mathrm{~cm}^{2}$ & 24 & 22,9 \\
\hline $2.5-5.0 \mathrm{~cm}^{2}$ & 14 & 13,3 \\
\hline $5.1-10.0 \mathrm{~cm}^{2}$ & 11 & 10,5 \\
\hline$>10 \mathrm{~cm}^{2}$ & 56 & 53,3 \\
\hline
\end{tabular}

superior incompleto (2,9\%). Quanto a renda, a maior parte dos participantes declarou receber até um salário mínimo (64,8\%), seguido de dois a três salários mínimos $(31,4 \%)$ e mais de três salários mínimos $(3,8 \%)$.

Quanto às características clínicas das lesões, a maioria se localizava na região do maléolo $(56,2 \%)$, com tempo de surgimento igual ou superior a 40 meses $(54,3 \%)$ e $62,9 \%$ não eram recidivas. Houve predominância de lesões maiores que $10 \mathrm{~cm}^{2}(53,3 \%)$, segundo o método de planimetria manual, que consiste na mensuração através do desenho do formato da ferida, utilizando folha de plástico transparente estéril, caneta retroprojetora e papel quadriculado em centímetros, fornecendo, assim, o tamanho da lesão com precisão ${ }^{8}$ (Tabela 2).

A maioria das lesões apresentava exsudato seroso $(91,4 \%)$ em pequena quantidade (40\%), com 51 a $75 \%$ de área granulada (36,2\%). Quanto à presença de edema, a maioria dos participantes apresentava edema sem cacifo ou firme (44,8\%) (Tabela 3$)$.

Tabela 3. Distribuição das úlceras conforme tipo e quantidade de exsudato, quantidade de tecido necrótico e tecido de granulação e tipo de edema de perna. Niterói, RJ, 2019. ( $N=105)$.

\begin{tabular}{lcr}
\hline \multicolumn{1}{c}{ Variável } & N & \% \\
\hline Tipo de Exsudato & & \\
\hline Serosanguinolento & 8 & 7,6 \\
\hline Seroso & 96 & 91,4 \\
\hline Seropurulento & 1 & 1,0 \\
\hline Quantidade de Exsudato & & \\
\hline Escassa & 22 & 21,0 \\
\hline Pequena & 42 & 40,0 \\
\hline Moderada & 28 & 26,7 \\
\hline Grande & 13 & 12,4 \\
\hline Quantidade de Tecido Necrótico & & \\
\hline Ausente & 29 & 27,6 \\
\hline 1 a 25\% coberta & 38 & 36,2 \\
\hline 26 a 50\% coberta & 21 & 20,0 \\
\hline 51 a 75\% coberta & 4 & 3,8 \\
\hline 76 a 100\% coberta & 13 & 12,4 \\
\hline Quantidade de Tecido de granulação & & \\
\hline 76 a 100\% coberta & 29 & 27,6 \\
\hline 51 a 75\% coberta & 38 & 36,2 \\
\hline 26 a 50\% coberta & 21 & 20,0 \\
\hline 1 a 25\% coberta & 17 & 16,2 \\
\hline Tipo de edema de perna & & \\
\hline Nenhum & 28 & 26,7 \\
\hline Sem cacifo ou firme & 47 & 44,8 \\
\hline Com cacifo & 8 & 7,6 \\
\hline Fibrose & 21 & 20,0 \\
\hline Endurecida & 1,0 \\
\hline
\end{tabular}


Tabela 4. Avaliação da intensidade e frequência da dor e qualidade de vida. Niterói, RJ, 2019. ( $=105)$.

\begin{tabular}{lcc}
\hline \multicolumn{1}{c}{ Variável } & N & \% \\
\hline Intensidade da Dor & & \\
\hline Nenhuma & 32 & 30,5 \\
\hline$>0-2$ & 11 & 10,5 \\
\hline $2-4$ & 18 & 17,1 \\
$>4-7$ & 13 & 12,4 \\
\hline$>7$ & 31 & 29,5 \\
\hline Frequência da Dor & & \\
\hline Nenhuma & 32 & 30,5 \\
\hline Ocasional & 31 & 29,5 \\
\hline Depende da posição & 33 & 31,4 \\
\hline Constante & 8 & 7,6 \\
\hline Prejudica o sono & 1 & 1,0 \\
\hline Qualidade de Vida & & \\
\hline Muito satisfeito & 1 & 1,0 \\
\hline Satisfeito & 47 & 44,8 \\
\hline Pouco satisfeito & 22 & 21,0 \\
\hline Insatisfeito & 25 & 23,8 \\
\hline Péssimo & 10 & 9,5 \\
\hline
\end{tabular}

Quanto à frequência de dor, a maioria dos participantes declarou sentir dor dependendo da posição do membro $(31,4 \%)$, de intensidade $>7(29,5 \%)$ em escala numérica. Em relação à qualidade de vida a maioria referiu se sentir satisfeito $(44,8 \%)$ (Tabela 4).

\section{DISCUSSÃO}

Alguns estudos revelam predominância de lesões em pacientes do sexo masculino, ${ }^{2,9-11}$ enquanto outros mencionam maior número no sexo feminino., ${ }^{8,12,13}$ Há descrição na literatura que a diferença percentual entre os sexos feminino e masculino de indivíduos acometidos por feridas crônicas vem diminuindo ao longo dos anos. ${ }^{3,13}$

Os resultados referentes a idade dos participantes condizem com o fato da maioria das feridas crônicas estar associada às condições da população idosa, incluindo maior prevalência de doenças crônico-degenerativas, além do efeito prejudicial do envelhecimento na cicatrização e mecanismos de reparo das lesões. ${ }^{3,5,5,14}$ Com o avanço da idade, há uma diminuição da resposta inflamatória, reduzindo o metabolismo do colágeno, a angiogênese e a epitelização, tornando a cicatrização mais lenta nos idosos. ${ }^{5}$

A predominância de baixo nível de escolaridade é um achado importante a ser considerado para o manejo do cuidado de enfermagem, uma vez que o tratamento das lesões envolve ações tanto farmacológicas quanto educativas, objetivando controle da causa subjacente da ulceração e dos fatores que a exacerbam, para promover a cicatrização e a prevenção de recidiva. ${ }^{15} \mathrm{As}$ orientações fornecidas pelos profissionais de saúde podem ser menos facilmente compreendidas por pacientes com menor nível de instrução, dessa forma, as ações educativas devem ser adaptadas e direcionadas de forma individualizada, possibilitando ao paciente o entendimento e aplicação das orientações na sua rotina. ${ }^{11}$

Os resultados desta pesquisa para as variáveis escolaridade e renda são semelhantes a outros estudos feitos com pacientes com feridas crônicas e podem ser explicados devido aos campos de pesquisa pertencerem à rede pública de saúde, apresentando uma clientela com menor renda e menos anos de estudo. ${ }^{3,5,16,17}$ Esses fatores podem estar associados com um estilo de vida que favoreça o surgimento de úlceras, com dificuldade de acesso a serviços de saúde especializados e manejo inadequado das lesões. ${ }^{5} \mathrm{~A}$ renda é um dado importante a ser considerado no contexto das feridas crônicas, pois a ausência de recursos financeiros para manutenção do tratamento pode acarretar o abandono da terapia pelo paciente. ${ }^{3}$

A insuficiência venosa crônica é responsável por cerca de 70\% das úlceras das pernas, com prevalência geral na população de 1 a 2\%. ${ }^{15}$ Está relacionada à hipertensão venosa prolongada, podendo ser resultado da existência de varizes primárias, sequela de trombose profunda, anomalias valvulares venosas ou outras causas capazes de interferir no retorno do sangue venoso. ${ }^{5} \mathrm{~A}$ hipertensão arterial sistêmica e diabetes também foram frequentes como comorbidades presentes e confirmam dados presentes na literatura que associam a maioria das feridas crônicas com essas condições, uma vez que interferem no processo de cicatrização devido a complicações vasculares. ${ }^{2-4}$

A maioria das lesões avaliadas foi de etiologia venosa, sendo esse resultado compatível com dados da literatura que afirmam que as úlceras venosas se destacam entre as úlceras de perna, representando de 70 a $90 \%$ de todas as úlceras de membros inferiores e são consideradas a complicação mais grave da insuficiência venosa crônica, sendo causadas essencialmente por hipertensão venosa. ${ }^{4,5,15}$ As úlceras diabéticas e possíveis amputações são as complicações mais graves do diabetes mellitus e ocorrem em cerca de $15 \%$ a $25 \%$ dos pacientes com diabetes, como consequência da evolução da neuropatia. ${ }^{8,18}$

A predominância de lesões na região maleolar se explica pelo fato da maioria das úlceras de perna presentes neste estudo ser de etiologia venosa, as quais predominantemente localizam-se na porção distal dos membros inferiores, principalmente na região do maléolo medial. ${ }^{4,8,11,13}$

O tempo prolongado de cicatrização é previsto, devido a fisiopatologia das feridas crônicas e estes resultados são semelhantes aos de outros estudos realizados, evidenciando que a maioria dos pacientes possui úlcera de perna durante anos, podendo haver recidivas ou não. ${ }^{5,11,13-15} \mathrm{Em}$ relação ao tamanho, não há consenso na literatura sobre a classificação 
das úlceras como pequenas, médias e grandes, havendo divergências entre estudos. ${ }^{13}$

Quanto ao tipo de exsudato, a predominância do tipo seroso se justifica pelo fato das úlceras venosas, presentes em maior número neste estudo, apresentarem mais comumente exsudato do tipo seroso e em pequena quantidade, sendo achados compatíveis com dados de outros estudos semelhantes com feridas crônicas. ${ }^{4,8,11}$

A característica do tecido no leito da ferida é um importante indicador do estágio da cicatrização alcançado ou de complicações que possam estar presentes. ${ }^{3,4}$ Os resultados encontrados evidenciam que a maioria das lesões apresentava um prognóstico favorável, com pouca ou nenhuma área de necrose e predominância do tecido granulado, caracterizado como o estágio da cicatrização que antecede a epitelização. ${ }^{3,8}$

O edema é um fator comum entre pacientes com úlceras de perna, conforme dados encontrados também em outros estudos. ${ }^{8,11,13}$ A insuficiência venosa, comorbidade destacada nesta pesquisa, está envolvida com a hipertensão venosa, que danifica os capilares tornando-os mais permeáveis, permitindo a passagem de diversas moléculas, gerando eczema, causado pela degradação da hemoglobina; edema, devido ao acúmulo de líquido intersticial, hiperpigmentação e lipodermatosclerose. ${ }^{17}$

Os resultados referentes a presença de dor corroboram evidências de que a dor é um dos principais problemas que acometem os indivíduos com úlceras de perna, podendo estar presente em cerca de 28 a $65 \%$ das pessoas com lesões e apresentar-se com diferentes características, independentemente do tamanho da ferida. ${ }^{11,13,14}$ A condição de piora da dor dependendo da posição condiz com o panorama predominante das úlceras venosas, que possuem como característica a dor que agrava ao ficar de pé ou andar e é aliviada com elevação dos membros. ${ }^{13}$ Esses achados reforçam a importância da incorporação da avaliação da dor pelos profissionais de saúde na rotina de cuidados com essa população, além das orientações relevantes. ${ }^{11}$

Estudos prévios sobre a qualidade de vida de indivíduos com úlceras de perna associaram os domínios relacionados à capacidade funcional, dor e limitação por aspectos físicos e emocionais como os responsáveis por prejudicar o padrão de qualidade de vida. Já os domínios relacionados com escores maiores foram os relacionados ao estado geral de saúde, vitalidade, aspectos sociais e saúde mental. ${ }^{11}$ Dessa forma, esses resultados sugerem que apesar do impacto das lesões crônicas na vida do indivíduo, alguns fatores têm importante contribuição positiva no que diz respeito à percepção do paciente sobre sua qualidade de vida.

\section{CONCLUSÃO E IMPLICAÇÕES PARA A PRÁTICA}

Em relação às características sociodemográficas, evidenciou-se que a maioria dos pacientes pertencia ao sexo masculino, na faixa de 60 a 80 anos, com ensino fundamental incompleto e renda declarada de até um salário mínimo. As comorbidades mais frequentes foram insuficiência venosa crônica e hipertensão arterial sistêmica. A maioria das lesões foi de etiologia venosa, com localização na região maleolar, com surgimento por tempo igual ou superior a 40 meses, não recidivantes, de tamanho maior que $10 \mathrm{~cm}^{2}$, apresentando exsudato seroso em pequena quantidade, com pouca ou nenhuma área de necrose e com predominância de tecido granulado. O edema sem cacifo ou firme foi predominante, a maioria dos participantes declarou sentir dor intensa (>7) relacionada com a posição do membro e, apesar desses relatos, a maior parte referiu se sentir satisfeito em relação a qualidade de vida.

O conhecimento do perfil da população acompanhada e dos dados clínicos das feridas crônicas possibilitam uma atuação mais direcionada e individualizada da equipe de enfermagem durante a assistência ambulatorial, auxiliando a determinação de cuidados específicos conforme as características predominantes da população e das lesões, visando a otimização do tratamento com orientações e atuações adaptadas às necessidades e especificidades da clientela atendida.

Como limitação do estudo destaca-se o tamanho amostral, pois embora todos os participantes disponíveis nos cenários tenham sido incluídos, durante o período de coleta houve na unidade hospitalar uma interrupção do fluxo de admissão de pacientes encaminhados de unidades externas, impossibilitando, assim, que novos pacientes iniciassem tratamento no ambulatório, e o outro cenário se tratava de uma policlínica regional, com atendimento restrito a população local.

\section{FINANCIAMENTO}

Fundação de Amparo à Pesquisa do Estado do Rio de Janeiro (FAPERJ), à pesquisa com o título de "Análise do microbioma de feridas crônicas infectadas e colonizadas e relação da microbiota da pele no processo de cicatrização", coordenado por Beatriz Guitton Renaud Baptista de Oliveira. Processo número E-26/010.101008/2018.

\section{CONTRIBUIÇÕES DOS AUTORES}

Desenho do estudo. Coleta ou produção dos dados. Análise de dados e interpretação dos resultados. Redação e revisão crítica do manuscrito. Aprovação da versão final do artigo. Responsabilidade por todos os aspectos do conteúdo e a integridade do artigo publicado. Fernanda Rabello Sergio. Isabelle Andrade Silveira. Beatriz Guitton Renaud Baptista de Oliveira

\section{EDITOR ASSOCIADO:}

Cristina Rosa Baixinho

\section{REFERÊNCIAS}

1. Rabeh SAN, Gonçalves MBB, Carili MHL, Nogueira PC, Miyazaki MY Construction and validation of a virtual educational module for topica treatment of chronic wounds. Rev Enferm UERJ [Internet]. $2012 \mathrm{dez}$ [citado 2020 maio 1];20:603-8. Disponível em: https://www.e-publicacoes. uerj.br/index.php/enfermagemuerj/article/view/5819/4229 
2. Squizatto RH, Braz RM, Lopes AO, Rafaldini BP, Almeida DB, Poletti NAA. Profile of users attended at a wound care outpatient clinic. Cogitare Enferm. 2017 jan;22(1):1-9. http://dx.doi.org/10.5380/ce.v22i1.48472.

3. Vieira CPB, Furtado AS, Almeida PCD, Luz MHBA, Pereira AFM Prevalence and characterization of chronic wounds in elderly persons assisted in primary care. Rev Baiana Enferm. 2017 ago;31(3):e17397. http://dx.doi.org/10.18471/rbe.v31i3.17397.

4. Oliveira BGRB, Castro JBA, Granjeiro JM. Epidemiologic and clinical overview of patients with chronic wounds treated at ambulatory. Rev Enferm UERJ [Internet]. 2013 dez; [citado 2020 maio 1];21:612-7. Disponível em: https://www.e-publicacoes.uerj.br/index.php/enfermagemuerj/ article/view/10035/7820

5. Medeiros ABA, Frazão CMFQ, Fernandes MICD, Andriola IC, Lopes MVO, Lira ALBC. Association of socioeconomic and clinical factors and tissue integrity outcome in patients with ulcers. Rev Gaúcha Enferm. 2016 mar;37(1):e54105. http://dx.doi.org/10.1590/1983-1447.2016.01.54105. PMid:26934612.

6. Morton LM, Phillips TJ. Wound healing and treating wounds: differential diagnosis and evaluation of chronic wounds. J Am Acad Dermatol. 2016 abr;74(4):589-605. http://dx.doi.org/10.1016/j.jaad.2015.08.068. PMid:26979352.

7. Silveira IA, Oliveira BGRB, Souza PA, Santana RF, Carvalho MR. Crosscultural adaptation of the Leg Ulcer Measurement Tool for Brazil: nursing methodology research. Rev Bras Enferm. 2020 jun;73(4):e20180944 http://dx.doi.org/10.1590/0034-7167-2018-0944. PMid:32578732.

8. Pessanha FS, Oliveira BGRB. Sociodemographic and clinical profile of ambulatory patients with leg ulcers. Rev. Enferm. UFPE Online. 2015 jul;9(7):8551-60. http://dx.doi.org/10.5205/reuol.7651-67144-1SM.0907201507.

9. Campoi ALM, Felicidade PJ, Martins LCN, Barbosa LBM, Alves GA, Ferreira LA. Nursing care for patients with chronic wounds: an experience report. REFACS. 2018 maio;7(2):248-55. http://dx.doi.org/10.18554/ refacs.v7i2.3045.
10. Resende NM, Nascimento TC, Lopes FRF, Prates Jr AG, Souza NM Care of people with chronic wounds in Primary Health Care. J Manag Prim Heal Care. 2017;8(1):99-108. http://dx.doi.org/10.14295/jmphc. v8i1.271.

11. Tavares APC, Sá SPC, Oliveira BGRB, Sousa AI. Quality of life of elderly patients with leg ulcers. Esc Anna Nery. 2017;21(4):1-9. http://dx.doi. org/10.1590/2177-9465-ean-2017-0134.

12. Liedke DCF, Johann DA, Danski MTR. A nursing consultation for treatment of wounds in a teaching hospital. Cogitare Enferm. 2014 jul/ set;19(3):590-6. http://dx.doi.org/10.5380/ce.v19i3.34486.

13. Silveira IA, Oliveira BGRB, Oliveira AP. Pain pattern in patients with leg ulcers. Rev Enferm UFPE. 2017 fev; 11(2):617-24.

14. Tavares APC, Pereira E S, Sá SPC. Impact of leg ulcer on quality of life of elderly: an integrative review. Revista Enfermagem Atual In Derme [Internet]. 2019 abr; [citado 2020 maio 1];78(16):49-55. Disponíve em: https://revistaenfermagematual.com.br/index.php/revista/article/ view/360/243

15. Benevides JL, Coutinho JFV, Pascoal LC, Joventino ES, Martins MC Gubert FA et al. Development and validation of educational technology for venous ulcer care. Rev Esc Enferm USP. 2016;50(2):309-12. http:// dx.doi.org/10.1590/S0080-623420160000200018. PMid:27384212.

16. Barros MPL, Ferreira PJO, Maniva SJCF, Holanda RE. Characterization of chronic wounds of a group of patients treated at home. R. Interd [Internet]. 2016; [citado 2020 maio 1];9(3):1-11. Disponível em: https:// revistainterdisciplinar.uninovafapi.edu.br/index.php/revinter/article/ view/926/pdf_333

17. Oliveira SB, Soares DA, Pires OS. Prevalence of venous ulcers and associated factors among adults of a health center in Vitória da Conquista - BA. J. Res. Fundam. Care. 2015 jul;7(3):2659-69. http:// dx.doi.org/10.9789/2175-5361.2015.v7i3.2659-2669.

18. Oliveira BGRB, Silva JA, Silveira IA, Santos NC, Carvalho MR. Clinical evaluation instruments for leg ulcers. Rev. Enferm. Atual In Derme [Internet]. 2019; [citado 2020 maio 1];9(spe):87. Disponível em: https:// revistaenfermagematual.com/index.php/revista/article/view/171/73 